\title{
IMPACTOS DA IRRIGAÇÃO NA VARIABILIDADE ESPACIAL E TEMPORAL DA SALINIDADE DE UM SOLO ALUVIAL NO SEMI-ÁRIDO POTIGUAR
}

\author{
Djeson Mateus Alves da Costa \\ Professor do Departamento de Formação de Professores, CEFET-RN, Doutor em \\ Engenharia Química. \\ djeson@cefetrn.br
}

\begin{abstract}
RESUMO
Este trabalho teve por objetivo estudar a variabilidade espacial e temporal da salinidade de um solo aluvial de três áreas agrícolas, com aproximadamente 40,5 ha. Uma delas nunca foi utilizada com cultivo irrigado $\left(\mathrm{A}_{1}\right)$, outra há pouco tempo tem sido utilizada para esse fim $\left(\mathrm{A}_{2}\right)$, enquanto a terceira já serviu $\left(\mathrm{A}_{3}\right)$, há mais de quinze anos, para essa atividade agrícola. Coletaram-se amostras de solo nas camadas de 0-30, 30-60 e 60-90 cm, numa área localizada às margens do Rio Cabugí, no Município de Afonso Bezerra-RN, utilizando-se um sistema numa malha regular de 100x100 m. Avaliou-se o potencial de hidrogênio iônico na pasta saturada $(\mathrm{pH})$, a condutividade elétrica do extrato saturado (CE) e a porcentagem de sódio trocável do solo (PST), nas três áreas e nas respectivas camadas. Verificou-se baixo coeficiente de variação para o $\mathrm{pH}$ e alto para a CE e PST. A prática da irrigação proporcionou aumento significativo na variabilidade espacial e temporal da CE e da PST do solo ao mesmo tempo em que o nível de profundidade da camada, na qual se fez a coleta de amostra de solo, não afetou essas variáveis $(\mathrm{P} \leq 0,05)$. Os mapas de isolinhas, para a CE e a PST, permitiram identificar os setores com maiores riscos à afetação por sais.
\end{abstract}

Palavras-chave: condutividade elétrica, sódio trocável, hidrogênio iônico.

\section{IMPACTS OF IRRIGATION IN SPATIAL AND TEMPORAL VARIABILITY OF SALINITY IN ALLUVIAL SOIL OF THE POTIGUAR SEMI-ARID}

\begin{abstract}
Aims this work was to investigate the spatial and temporal variability of salinity of the alluvial soil of three agricultural areas, with 40.5 ha approximately. One of them never was utilized in irrigation $\left(A_{1}\right)$, another was utilized by short time for this practice $\left(A_{2}\right)$, while the third area $\left(\mathrm{A}_{3}\right)$ was utilized for more than fifteen years in this activity. Samples of soil were made in layers of 0-30, 30-60 and 60-90 cm, in an area localized in the Cabugí Ríver margins, in Afonso Bezerra-RN Council, following a regular system in a 100x100 m mesh. The potential ionic hydrogen in the saturated soil $(\mathrm{pH})$, the electric conductivity of saturated extract (EC) and exchangeable sodium percentage of the soil (ESP) were evaluated in three areas and layers respectively. Was observed coefficient of variation low for the $\mathrm{pH}$ and high for the EC and ESP. The practice of irrigation increased the spatial and temporal variability of the EC and ESP in the soil while the level of the layer didn't effect these variables $(\mathrm{P} \leq 0.05)$. The maps of isohypsas, for EC and ESP, helped in the identification of the sectors with salinity risks.
\end{abstract}

Key-words: Salinity, exchangeable sodium, spatial and temporal variability. 


\section{IMPACTOS DA IRRIGAÇÃO NA VARIABILIDADE ESPACIAL E TEMPORAL DA SALINIDADE DE UM SOLO ALUVIAL NO SEMI-ÁRIDO POTIGUAR}

\section{INTRODUÇÃO}

A irrigação é um manejo que contribui para o aumento da produção agrícola e da produtividade das plantas, porém quando má conduzida proporciona aumento da salinidade do solo, induzindo à redução do potencial produtivo do sistema solo-planta. Esse efeito negativo é mais intenso nas regiões áridas e semi-áridas devido às altas taxas de evaporação da água do solo e as baixas precipitações, o que ocasiona a precipitação dos sais solúveis em seus solos. Esse fenômeno diminui o potencial hídrico do solo, reduzindo a absorção de água pelas plantas e, consequentemente, sua produtividade (OLIVEIRA, 1997). As condições do solo, do clima, do lençol freático e de irrigação, associadas às atividades antrópicas podem acelerar, ainda mais, esse fenômeno imprimindo uma maior variabilidade ao solo (ALBUQUERQUE et al., 1996).

A avaliação do desempenho da irrigação pode ser feita por meio da caracterização espacial das propriedades físicas e químicas do solo. Estudos que objetivam dimensionar a variabilidade espacial dos principais parâmetros do solo, dentro de uma unidade de solo, ainda são pouco relacionados no Brasil (CORRÊA, 1986). Algumas áreas aparentemente homogêneas podem apresentar grande variabilidade espacial, seja qual for o parâmetro analisado (NIELSEN et al., 1973).

A variabilidade para alguns atributos do solo, expressa pelo coeficiente de variação (CV), pode apresentar valores inferiores a $10 \%$, enquanto, para outros, pode ser superiores a $1000 \%$. Dessa forma, a classificação para a variabilidade pode ser baixa (CV $\leq 12 \%)$, média $(12 \%<\mathrm{CV} \leq 52 \%)$ e alta $(\mathrm{CV}>52 \%)$, conforme Warrick \& Nielsen (1980). Esse método não considera as posições geográficas relativas de cada amostra de solo analisada, admitindo homogeneidade da área e independência entre as amostras, o que não pode ser aplicado para algumas áreas em estudo.

Os solos aluviais são intensamente utilizados para a prática da agricultura irrigada, principalmente devido suas características físicas e químicas e sua proximidade das fontes hídricas. Por isso, é muito importante ter o conhecimento da variabilidade espacial desses solos, seja em superfície ou em profundidade, pois de posse dessas informações é possível planejar as técnicas adequadas para o manejo agrícola. Segundo Trangmar et al. (1985), algumas propriedades alteradas pelo manejo apresentam maior variabilidade espacial do que aquelas associadas às características genéticas do solo.

Nesse contexto, a geoestatística é aplicada aos fenômenos que flutuam no tempo e/ou no espaço oferecendo ferramentas determinísticas e estatísticas que possibilitem entender e modelar a variabilidade espacial de uma determinada propriedade (DEUTSCH \& JOURNEL, 1998). A construção de mapas de contorno - isolinhas ou curvas de níveis - e o delineamento de espaçamento e disposição de amostras no campo são outras aplicações imediatas que auxiliam na identificação dessa variabilidade (FREITAS, 2000).

O objetivo deste trabalho foi avaliar a variabilidade espacial e temporal da salinidade do solo em três áreas agrícolas, (uma que nunca foi utilizada com irrigação, outra que há 
pouco tempo tem sido utilizada com irrigação, e uma terceira que há mais de quinze tem sido irrigada e plantadas com diferentes culturas), e correlacionar os resultados obtidos.

\section{MATERIAL E MÉTODOS}

O trabalho foi realizado no período de outubro a dezembro de 2000 numa área localizada à margem esquerda do Rio Cabugí (5 $5^{\circ} 28^{\prime} 27,53^{\prime \prime}$ Sul; 36 31'51,52" Oeste; altitude de $48 \mathrm{~m}$ em relação ao nível do mar), pertencente ao perímetro irrigado do Município de Afonso Bezerra-RN. A área estudada abrange aproximadamente 40,5 ha., sendo constituída de solo de aluvião, classificado como Neossolo Flúvico Distrófico (EMBRAPA, 2006), apresentando no triângulo textural a classificação areia em alguns setores. A classificação climática para a região, segundo Köppen (1948), é do tipo Bsh, com precipitação total anual média de $516,5 \mathrm{~mm}$, temperatura média de $27^{\circ} \mathrm{C}$ e umidade relativa média anual de $70 \%$ (IDEMA, 1999).

A área pesquisada foi dividida em três sub-áreas ou setores, recebendo as seguintes denominações: $A_{1}$ - área nunca irrigada; $A_{2}$ - área há 4 anos irrigada; e, $A_{3}$ - área irrigada a mais de 15 anos.

As amostras de solo foram coletadas com um trado manual (caixa de coleta 9,0x30 cm) em 36 pontos da área (12 pontos por setor), nas camadas de 0-30 cm, 30-60 cm e 60-90 cm, perfazendo um total de 108 amostras, num sistema em malha uniforme de 100x100 m. Utilizou-se um GPS (Global Positioning System) para o georreferenciamento, no sistema UTM (Universal Transversa de Mercator), dos pontos de coleta das amostras de solo e, a partir desses, interpolou-se os dados e geraram-se os mapas de isolinhas.

As variabilidades espacial e temporal foram avaliadas por meio da significância das alterações nas magnitudes das variáveis, aplicados a cada sub-área e profundidade das camadas do solo e da construção de mapas de isolinhas, associando-os ao tempo de aplicação da irrigação. Na confecção desses mapas, utilizando o algoritmo da krigagem, adotou-se o software SURFER Versão 8.0 (2002). Os parâmetros avaliados foram o pH na pasta saturada, a condutividade elétrica do extrato saturado (CE) e a porcentagem de sódio trocável (PST), seguindo as metodologias de análises de solo da EMBRAPA (1997).

Os dados foram ponderados por meio da estatística descritiva, baseando-se nas seguintes medidas: média, mediana, valores máximo e mínimo, coeficiente de variação, desvio padrão, amplitude total e análise de população. Utilizou-se o teste de normalidade de Kolmogorov-Smirnov (KS). A análise de variância foi feita pelo teste $\mathrm{F}$ e as médias comparadas pelo teste de Tukey, ambas a 1\% e 5\% de probabilidade, com a utilização do software ASSISTAT Versão beta 7.5 (2008).

\section{RESULTADOS E DISCUSSÃO}

\section{Estatística descritiva}

A análise de variância indicou que a prática da irrigação não afetou o $\mathrm{pH}$ do extrato saturado do solo $(\mathrm{P} \leq 0,05)$, ao mesmo tempo em que proporcionou aumentos nos valores da $\mathrm{CE}$ do extrato saturado e da PST do solo ( $\mathrm{P} \leq 0,01)$, sendo que esse manejo foi mais efetivo, à esses acréscimos, quanto mais longo foi o tempo de exposição do solo a essa atividade 
agrícola, fato esse também observado por Costa et al. (2004) e Mendes et al. (2008). Por outro lado, com o aumento na profundidade da camada do solo não se detectou variações significativas para essas variáveis, em todas as sub-áreas objetos do estudo $(\mathrm{P} \leq 0,05)$, a exceção do $\mathrm{pH}$ em $\mathrm{A}_{3}$ cujo valor médio para a camada próxima a superfície $(7,39)$ foi significativamente maior $(\mathrm{P} \leq 0,05)$ que ao da camada adjacente $(7,09)$.

$\mathrm{O}$ valor médio para o $\mathrm{pH}$ do extrato saturado do solo nos diferentes setores e nas respectivas camadas foi igual a 7,11, sendo que essa variável apresentou baixo coeficiente de variação $(C V=5,67 \%)$, segundo critérios apresentados por Warrick \& Nielsen (1980). A baixa amplitude verificada para o $\mathrm{pH}$ do solo nos diferentes setores, calculada pela diferença entre os limites mínimo $(6,22)$ e máximo $(7,84)$ e a proximidade entre a média e a mediana $(7,10)$ indicam uma tendência à distribuição normal, o que foi constatado em $\mathrm{A}_{3}$ por meio do teste de Kolmogorov-Smirnov $(\mathrm{P} \leq 0,05)$.

O maior valor da $\mathrm{CE}$ média do extrato saturado do solo no setor há muito tempo irrigado, $\mathrm{A}_{3}$, em relação aos valores observados nas outras sub-áreas, $\mathrm{A}_{1}$ e $\mathrm{A}_{2}$ (Tabela 1), pode ser uma indicação da utilização de água de má qualidade na irrigação. Devido às elevadas temperaturas e altas taxas de evapotranspiração predominantes na região é possível que os sais solúveis existentes nas águas disponíveis para a irrigação tenham se precipitados ao longo do perfil do solo, ocasionando esse aumento da salinidade, nas respectivas sub-áreas irrigadas (COSTA, 2001). No entanto, essa salinização depende também das características físicas e químicas do solo, das técnicas de manejo aplicadas (SILVA FILHO et al., 2000), da profundidade do lençol freático, da intensidade de chuvas e de tantos outros fatores hidrogeológicos (D'ALMEIDA et al. 2005).

Constatou-se, portanto, que a área pesquisada não apresentava problema de salinidade, pois

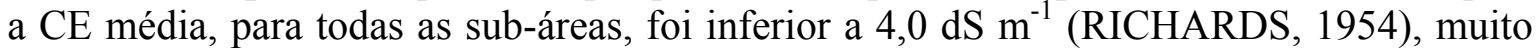
embora, nos setores irrigados já apresentem sintomas de aumento da salinidade, proporcionando maior variabilidade da salinidade do solo (ALBUQUERQUE et al., 1996), ratificada pelo alto coeficiente de variação, amplitude total e desvio padrão (Tabela 1).

Os dados apresentados na Tabela 1 indicam altos coeficientes de variação para a CE do extrato saturado e para a PST do solo, nas respectivas sub-áreas diagnosticadas, inclusive naquela onde nunca se fez irrigação, segundo critérios adotados por Warrick \& Nielsen (1980). Esta ocorrência é típica dos solos aluvionares, e provavelmente está relacionada às particularidades da própria gênese desse solo.

Por outro lado, os aumentos ocasionados na PST pode ser uma conseqüência do avanço gradual da sodicidade do solo das áreas irrigadas, devido ao uso de água salina, mesmo que esta apresente pouco teor de $\mathrm{Na}^{+}$(MILLS, 2003), cuja RAS desse recurso hídrico, determinada em outros trabalhos realizados por Costa (2001), apresentou valor médio igual a 5,25. Concomitantemente, as condições climáticas verificada para a região, nesse caso semi-árida, também devem ter contribuído para a precipitação de sais de sódio, ocasionando a elevação da PST nos setores há mais tempo irrigados (MACEDO \& MENINO, 1998). Por não ter sido observado variação significativa da PST no perfil do solo, pode-se inferir que o movimento ascendente e/ou descendente da água no solo, seja oriunda da irrigação ou da precipitação pluviométrica, proporcionou homogeneidade para a concentração de $\mathrm{Na}^{+}$nas diferentes camadas do solo. Segundo Souza et al. (2000), as características físicas do solo associadas às condições de drenagem podem também permitir o equilíbrio desse elemento químico, na zona radicular. 
Tabela 1: Medidas estatísticas do pH, da CE do extrato saturado e da PST do solo, na camada de 0 a $90 \mathrm{~cm}$, para as diferentes sub-áreas.

\begin{tabular}{lccr}
\hline Medida estatística & $\mathrm{pH}$ & $\mathrm{CE}\left(\mathrm{dS} \mathrm{m}^{-1}, 25^{\circ} \mathrm{C}\right)$ & PST (\%) \\
\hline Área nunca antes irrigada (A) & & & \\
Média & & 0,37 & 0,87 \\
Mediana & 7,00 & 0,24 & 0,65 \\
Máximo & 6,86 & 2,38 & 7,17 \\
Mínimo & 7,73 & 0,09 & 0,17 \\
Desvio padrão & 6,22 & 0,42 & 1,15 \\
Amplitude total & 0,45 & 2,29 & 7,00 \\
Coeficiente de variação (\%) & 1,51 & 112,28 & 130,66 \\
Distribuição & 6,59 & $\mathrm{DA}$ & $\mathrm{DA}$
\end{tabular}

\section{Área recentemente irrigada $\left(\mathrm{A}_{2}\right)$}

$\begin{array}{lccr}\text { Média } & 7,15 & 0,79 & 2,43 \\ \text { Mediana } & 6,09 & 0,30 & 1,23 \\ \text { Máximo } & 7,84 & 5,40 & 14,99 \\ \text { Mínimo } & 6,48 & 0,09 & 0,70 \\ \text { Desvio padrão } & 0,43 & 1,25 & 2,97 \\ \text { Amplitude total } & 1,36 & 5,31 & 14,29 \\ \text { Coeficiente de variação (\%) } & 6,09 & 158,11 & 123,14 \\ \text { Distribuição } & \text { DA } & \text { DA } & \text { DA }\end{array}$

Área há muito tempo irrigada $\left(\mathbf{A}_{3}\right)$

$\begin{array}{lcrr}\text { Média } & 7,19 & 1,98 & 6,36 \\ \text { Mediana } & 7,20 & 0,36 & 1,45 \\ \text { Máximo } & 7,77 & 12,78 & 24,09 \\ \text { Mínimo } & 6,53 & 0,13 & 0,34 \\ \text { Desvio padrão } & 0,32 & 2,99 & 8,09 \\ \text { Amplitude total } & 1,24 & 12,65 & 23,75 \\ \text { Coeficiente de variação (\%) } & 4,10 & 155,04 & 130,35 \\ \text { Distribuição } & \text { DS } & \text { DA } & \text { DA }\end{array}$

DS e DA: distribuição simétrica e assimétrica, respectivamente $(\mathrm{P} \leq 0,05)$.

Nesse caso, é provável que a permeabilidade, nos respectivos setores da área estudada, tenha sido adequada para que houvesse a lixiviação dos sais em todo o perfil do solo (FAGERIA et al., 1999), sem provocar a dispersão da argila. Todavia, Costa et al. (2004) observaram que a velocidade de infiltração da água em solos irrigados era inferior a encontrada em solos nunca antes irrigados, quando ambos apresentavam características homogêneas.

Dessa forma, os valores médios determinados para a PST do solo (Tabela 1) variaram na seguinte ordem crescente: área nunca antes irrigada $(0,87 \%)<$ área recentemente irrigada $(2,43 \%)<$ área há mais tempo irrigada $(6,36 \%)$. Portanto, os dados evidenciam que 
nenhum dos setores pesquisados $\left(\mathrm{A}_{1}, \mathrm{~A}_{2}\right.$ e $\left.\mathrm{A}_{3}\right)$ apresentou problemas de sodicidade, conforme classificação de Richards (1954).

\section{Análise da variabilidade espacial e temporal}

Apesar do $\mathrm{pH}$ do extrato saturado não ter apresentado valores médios significativamente diferentes nas diferentes sub-áreas, e em suas respectivas camadas, à exceção de $\mathrm{A}_{3}$ cujos valores variaram em função do nível da camada, observou-se incrementos na magnitude dessa variável, em todos os níveis, na área $\mathrm{A}_{3}$ (região central) em relação aos valores obtidos nas outras sub-áreas pesquisadas, $\mathrm{A}_{1}$ e $\mathrm{A}_{2}$ (regiões extremas à norte e à sul), conforme representado na Figura 1. Este fato pode está relacionado à maior intensidade de práticas de cultivo e de manejo do solo desenvolvidas na área a mais tempo irrigada principalmente na camada mais superficial do solo, comparada às das outras áreas, resultado que corrobora com as observações de Teixeira et al. (2003).

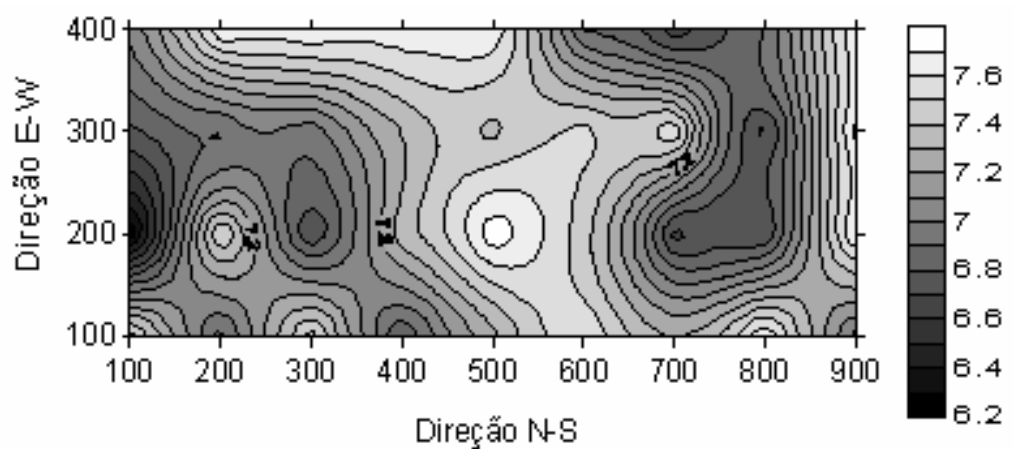

(A)

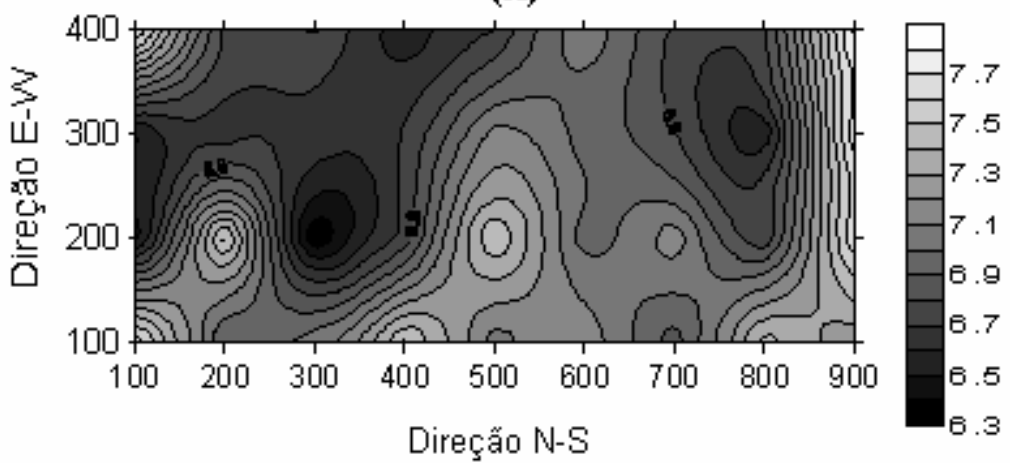

(B)

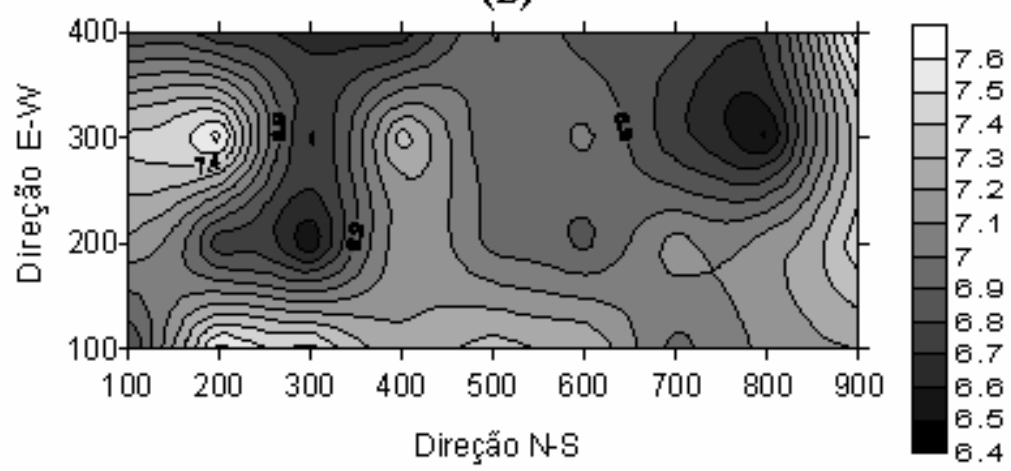

(C)

Figura 1: Mapas de isolinhas para o pH do solo da área experimental, nas camadas de 0 a $30 \mathrm{~cm}(\mathrm{~A})$, de 30 a $60 \mathrm{~cm}$ (B) e de 60 a $90 \mathrm{~cm}$ (C). 
$\mathrm{O}$ aumento da alcalinidade na sub-área $\mathrm{A}_{3}$ - setor central da Figura 1 - pode proporcionar redução na disponibilidade de nutrientes essenciais às plantas, ocasionando diminuição do potencial produtivo deste em relação ao dos demais setores (MENGEL et al., 2001).

Simultaneamente a concentração de sais, medida pela condutividade elétrica no extrato saturado (CE), e o valor da porcentagem de sódio trocável (PST) se apresentaram mais elevados na sub-área $A_{3}$ e mais baixos na $A_{1}$, caracterizando variabilidade espacial e temporal para essas variáveis, em razão do uso e do tempo de aplicação da irrigação (Figura 2). Portanto, os solos das sub-áreas $\mathrm{A}_{2}$ e $\mathrm{A}_{3}$, em ordem crescente, apresentaram maior risco à salinidade e sodicidade que os da sub-área $A_{1}$. Estas ressalvas condizem com as feitas por Costa et al. (2004) e Lopes et al. (2008), respectivamente, ao estudarem solos do perímetro irrigado na Bacia do Rio Cabugí-Afonso Bezerra-RN e na Bacia do AcaraúCE, no Brasil.

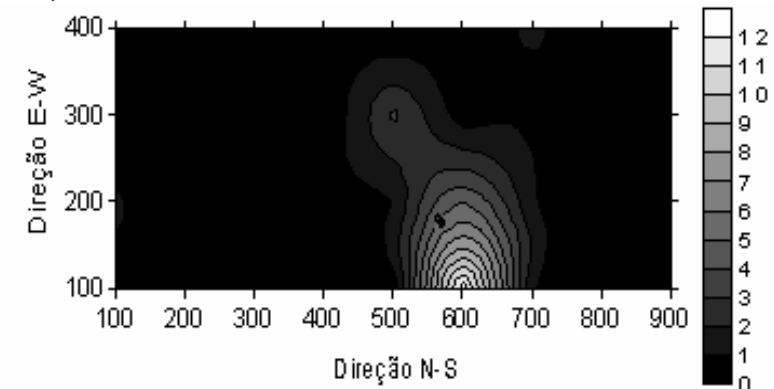

(A)

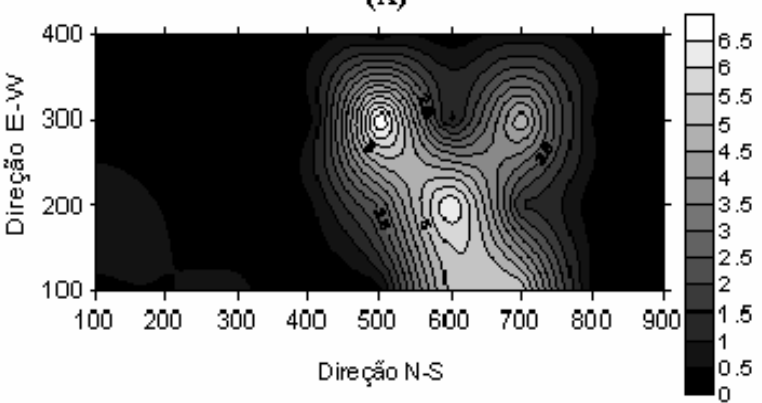

(B)

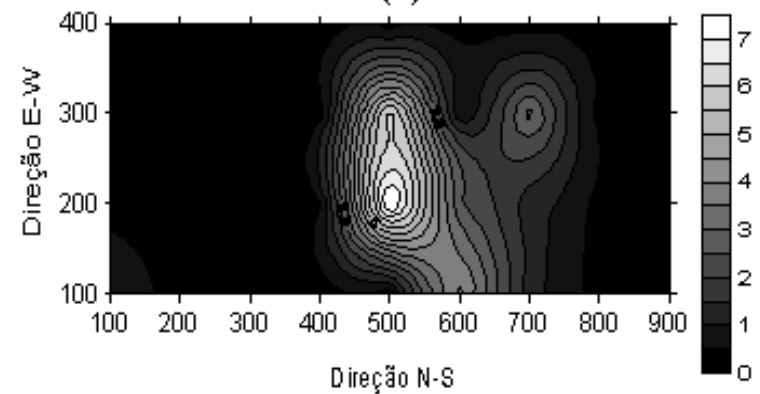

(C)

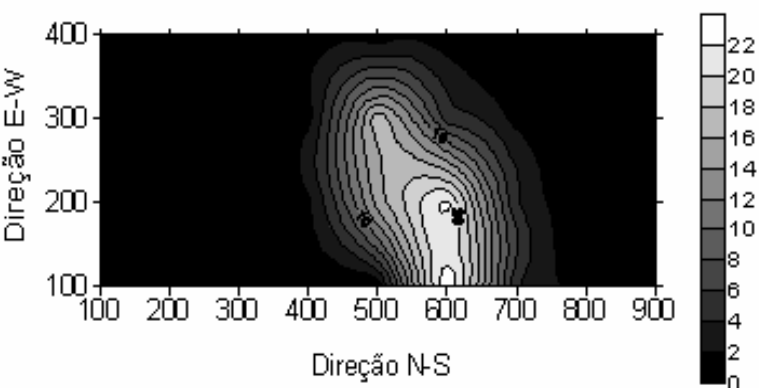

(D)

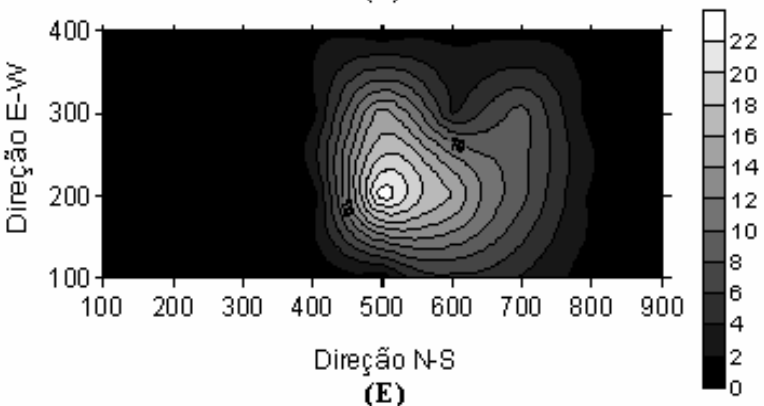

(E)

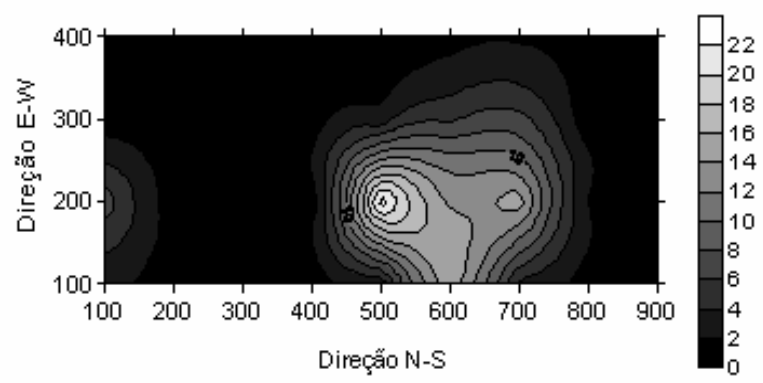

(F)

Figura 2: Mapas de isolinhas para a CE e para a PST do solo da área experimental, respectivamente, nas camadas de 0 a $30 \mathrm{~cm}$ (A e D), de 30 a $60 \mathrm{~cm}$ (B e E) e 60 a 90 cm (C e F).

O incremento nos valores da CE e da PST nas áreas submetidas a mais tempo à irrigação (área central da Figura 2), comparado com as magnitudes dessas variáveis na área nunca antes irrigada, foi detectado em todas as camada do perfil do solo avaliadas. 
Verificou-se, portanto, que a irrigação e o respectivo tempo de sua aplicação funcionaram como aceleradores do processo de degradação do solo, ocasionando maior variabilidade espacial e temporal às propriedades averiguadas, resultados que corroboram com as observações de D' Almeida et al. (2005). Essas constatações oferecem embasamentos para que se delineiem manejos adequados visando minimizar essa ação deteriorante, nas áreas com risco à afetação por sais, conforme comenta Zapata \& Playán (2000).

\section{CONCLUSÕES}

A maioria das variáveis diagnosticadas apresentou distribuição assimétrica em toda extensão e camadas da área pesquisada. Observou-se baixa variabilidade para o $\mathrm{pH}$ e elevada para a CE e PST do solo, nas respectivas sub-áreas.

A prática da irrigação e o tempo de sua aplicação não induziram variações nos valores do $\mathrm{pH}$ ao mesmo tempo em que proporcionaram aumentos nos da CE e da PST do solo, na camada de 0 a $90 \mathrm{~cm}$.

O solo é mais susceptível à afetação por sais com o uso e o aumento do tempo de irrigação.

\section{REFERÊNCIAS BIBLIOGRÁFICAS}

ALBUQUERQUE, J. A.; FIORIN, J. E.; REINERT, D. J.; MUTTI,L.S.M.; SILVA, E.P. da. Variabilidade espacial do solo e produção do milho. Revista Brasileira de Ciência do solo, Campinas, v.20, n.1, p.151-157, 1996.

CORREAA, J. B. D. Variabilidade espacial de características e propriedades físicas de latossolo roxo do Município de Lavras - MG. Escola Superior de Agricultura de Lavras, Lavras, 1986. 83p. (Dissertação de Mestrado).

COSTA, D. M. A. Caracterização Físico-Química de solos da Bacia do Rio Cabugí no Município de Afonso Bezerra-RN. Universidade Federal do Rio Grande do Norte, Natal, 2001. 122p. (Dissertação de Mestrado).

COSTA, D. M. A.; HOLANDA, J. S. \& FILHO, O. A. F. Caracterização de solos quanto a afetação por sais na bacia do Rio Cabugí - Afonso Bezerra-Rn. Holos, v.2, n.1, p.1-13, out., 2004.

D’ ALMEIDA, D. B. A.; ANDRADE, E. M.; MEIRELES, A. C. M. \& NESS, R. L. L. Importância relativa dos íons na salinidade de um Cambissolo na chapada do Apodi, Ceará. Engenharia Agrícola, v.25, n.3, p.615-621, 2005.

DEUTSCH, C. V. \& JOURNEL, A. G. GSLIB. Geostatistical Software Library and User's Guide. Oxford University Press, Second Edition, 1998. 369p.

EMPRESA BRASILEIRA DE PESQUISA AGROPECUÁRIA - EMBRAPA. Manual de métodos de análises de solo. 2.ed. Rio de Janeiro: EMBRAPA-CNPS, 1997. 212p. 
EMPRESA BRASILEIRA DE PESQUISA AGROPECUÁRIA - EMBRAPA. Sistema Brasileiro de Classificação de solos. Rio de Janeiro, 2006. 306p.

FAGERIA, N. K.; STONE, L. F. \& SANTOS, A. B. Maximização da eficiência de produção das culturas. Brasília, Embrapa Arroz e Feijão, 1999. 294p.

FREITAS, V. A. Análise de dados espaciais por meio de semivariogramas. Universidade Federal de Uberlândia, Uberlândia-MG, 2000. 34p.

INSTITUTO DE DESENVOLVIMENTO DO MEIO AMBIENTE - IDEMA-RN. Informativo Municipal de Afonso Bezerra-RN. Natal-RN, v.5, p.1, 1999.

KÖPPEN, W. Climatologia. Fondo de Cultura Econômica. México, 1948. 478p.

LOPES, J. F. B.; ANDRADE, E. M.; CHAVES, L. C. G. Impacto da irrigação sobre solos de perímetros irrigados na Bacia do Acaraú, Ceará, Brasil. Jaboticabal. Engenharia Agrícola, v.28, n.1, p.34-43, jan-mar., 2008.

MACEDO, L. S.; MENINO, I. B. Monitoramento de sais na água e nos solos irrigados do Projeto Vereda Grande, PB. Revista Brasileira de Engenharia Agrícola e Ambiental, v.2, n.1, p.262-267, 1998.

MENDES, J. S.; CHAVES, L. H. G.; CHAVES, I. B. Variabilidade temporal da fertilidade, salinidade e sodicidade de solos irrigados no município de Congo, PB. Revista Brasileira de Ciências Agrárias, v.3, n.1, p.13-19, jan-mar., 2008.

MENGEL, K.; KIRKBY, E. A.; KOSEGARTEN, H. APPEL, T. Principles of plant nutrition. 5. ed. Dordrecht: Kluwer Academic Publishers, 2001. 849p.

MILLS, B. Interpreting water analysis for crop and pasture. FS0334, Department of Primary Industries, State of Queensland, Brisbane, Australia, 2003.

NIELSEN, D. R.; BIGGAR, J. W. \& EHR, K. T. Spatial variability of field-measured soil water properties. Hilgardia, Berkley, v.42, n.7, p.215-259, 1973.

OLIVEIRA, M. de. Gênese, classificação e extensão de solos afetados por sais. In: GHEYI, H. R.; QUEIROZ, J. E.; MEDEIROS, J. M. (ed.) Manejo e controle da salinidade na agricultura irrigada. Campina Grande: UFPB/SBEA, 1997. p.1-37.

RICHARDS, L. A. Diagnosis and improvement of saline and alkaline soils. Washington. United States Salinity Laboratory Staff, 1954. 160p. (Agriculture Handbook, $60)$.

SILVA FILHO, S. B.; CAVAlCANTE, L. F.; OLIVEIRA, F. A.; LIMA, E. M. \& COSTA, J. R. M. Monitoramento da qualidade da água e acúmulo de sais no solo pela irrigação. Irriga, v.5, n.2, p.112-125, 2000.

SOUZA, L. C. de; QUEIROZ, J. E.; MEDEIROS, J. F.; GHEYI, H. R. Variabilidade espacial da salinidade de um solo aluvial no semi-árido paraibano. Revista Brasileira de Engenharia Agrícola e Ambiental, Campina Grande, v.4, n.2, p.30-40, jan-abr., 2000. 
TEIXEIRA, I. R; SOUZA, C. M.; BORÉM, A.; SILVA, G. F. Variação dos valores de pH e dos teores de carbono orgânico, cobre, manganês, zinco e ferro em profundidades em Argissolo Vermelho-Amarelo, sob diferentes sistemas de preparo do solo. Campinas, Bragantia, v.62, n.1, p.119-126, 2003.

TRANGMAR, B. B.; YOST, R. S. \& UEHARA, G. Application of geostatistics to spatial studies of soil properties. Advances Soil Science., 38:45-94,1985.

WARRICK, A. W. \& NIELSEN, D. R. Spatial variability of soil physical properties in the field. In: HILLEL, D., ed. Applications of soil physics. New York, Academic Press, 1980. cap.13, p.319-344.

ZAPATA, M. \& PLAYÁN, E. Elevation and infiltration in a level basin. I. Caracterizing variability. Irrigation Science. New York, v.19, n.4, p.155-164, Sept. 2000. 